ARTICLE

Received 2 Sep 2015 | Accepted 11 Dec 2015 | Published 4 Feb 2016

DOI: $10.1038 /$ ncomms10445

OPEN

\title{
Design of crystal-like aperiodic solids with selective disorder-phonon coupling
}

\author{
Alistair R. Overy ${ }^{1,2}$, Andrew B. Cairns ${ }^{1,3}$, Matthew J. Cliffe ${ }^{1,4}$, Arkadiy Simonov1, Matthew G. Tucker 2,5 \\ $\&$ Andrew L. Goodwin ${ }^{1}$
}

Functional materials design normally focuses on structurally ordered systems because disorder is considered detrimental to many functional properties. Here we challenge this paradigm by showing that particular types of strongly correlated disorder can give rise to useful characteristics that are inaccessible to ordered states. A judicious combination of low-symmetry building unit and high-symmetry topological template leads to aperiodic 'procrystalline' solids that harbour this type of disorder. We identify key classes of procrystalline states together with their characteristic diffraction behaviour, and establish mappings onto known and target materials. The strongly correlated disorder found in these systems is associated with specific sets of modulation periodicities distributed throughout the Brillouin zone. Lattice dynamical calculations reveal selective disorder-driven phonon broadening that resembles the poorly understood 'waterfall' effect observed in relaxor ferroelectrics. This property of procrystalline solids suggests a mechanism by which strongly correlated topological disorder might allow independently optimized thermal and electronic transport behaviour, such as required for high-performance thermoelectrics.

\footnotetext{
${ }^{1}$ Department of Chemistry, University of Oxford, Inorganic Chemistry Laboratory, South Parks Road, Oxford OX1 3QR, UK. ${ }^{2}$ Diamond Light Source, Chilton, Oxfordshire, OX11 ODE, UK. ${ }^{3}$ European Synchrotron Radiation Facility, 71 avenue des Martyrs, 38043 Grenoble, France. ${ }^{4}$ Department of Chemistry, University of Cambridge, Lensfield Road, Cambridge CB2 1EW, UK. ${ }^{5}$ ISIS Facility, Rutherford Appleton Laboratory, Harwell Oxford, Didcot, Oxfordshire OX11 OQX, UK. Correspondence and requests for materials should be addressed to A.L.G. (email: andrew.goodwin@chem.ox.ac.uk).
} 
T he relationship between building block geometry and bulk material structure is one of the cornerstones of structural science. By way of example, the solid phases of elemental $\mathrm{Xe}$ (ref. 1), $\mathrm{C}_{60}$ (ref. 2) and human adenovirus ${ }^{3}$ are all structurally related, not by virtue of any particular chemical similarity but because each of these phases reflects the same solution to the problem of packing weakly interacting spherical objects in threedimensional (3D) space. The reticular approach to understanding zeolite and metal-organic framework topologies is related from a conceptual viewpoint, because it links the geometries of moleculelike components (coordination polyhedra, molecular linkers) to the 3D architectures formed by their assemblies ${ }^{4,5}$. The importance of structure in determining the physical properties of solids is what then gives sense to the approach of developing new types of functional materials through informed design of constituent building blocks. It is precisely this type of 'ground-up' approach that has recently been exploited in the rational design of, for example, solid oxide fuel-cell cathodes and roomtemperature multiferroic candidates ${ }^{6,7}$.

To a large extent, the rich structural information accessible using crystallographic techniques has focussed effort on the design of crystalline materials. There are also obvious functional advantages to the long-range periodicity characteristic of crystals, because it governs useful correlated properties-including the lattice dynamics, electronic states, and charge, orbital and magnetic order. Moreover, crystal symmetry is central to mechanical properties such as piezoelectricity and ferroelasticity, and is clearly pivotal in determining phase transition behaviour. While certain building block geometries allow-or can even force ${ }^{8,9}$-non-crystalline assemblies, the link to function is usually much less clear in these cases. Indeed the received wisdom is that disorder is something to be avoided, despite increasingly strong empirical evidence that links disordered states to advanced functionalities ${ }^{10}$. So the development of disorderproperty relationships, and the eventual control over these properties through suitable building block design have emerged as key challenges in the field.

Here we develop an approach of intentionally designing functional disordered materials by focussing on systems in which structural disorder is extremely strongly correlated. The type of disorder we consider is similar to that found in ice, and our paper begins by developing a generalization of ice-like states to arbitrary materials geometries. We proceed to establish a link between the geometry of structural building blocks and the propensity for specific types of strongly correlated disorder in the resulting material assembly. This is the design element of our approach. Having made this connection, we suggest a number of physical realizations of these correlated disordered states. Our paper concludes by demonstrating how the correlated disorder deliberately engineered within one representative affects its lattice dynamics in a highly specific manner. This is the functional element of our approach because the effect we observe suggests, for example, a fundamentally new way of optimizing thermoelectric response.

\section{Results}

Generalization of ice rules. Our starting point is the simple toy model of square ice, in which water molecules are arranged on a square lattice and oriented so as to satisfy sensible hydrogenbonding rules (Fig. 1a). As there is no unique way of satisfying these rules the system is disordered, even if the molecule orientations are far from random. As in the real-world examples of cubic ice $\left(I_{c}\right)$ and its nano-confined variants ${ }^{11}$, this system is characterized by a degenerate manifold of structural ground states $^{12}$. An idea we will come to develop is that this propensity
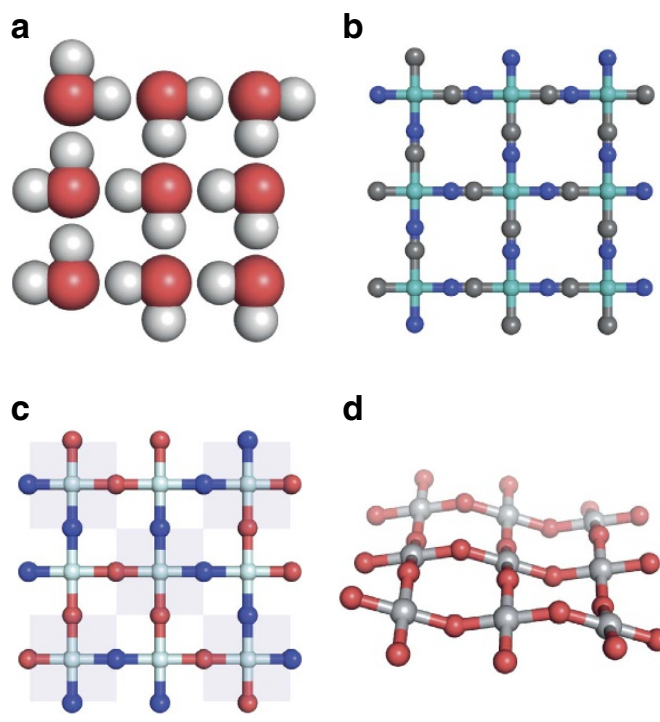

d

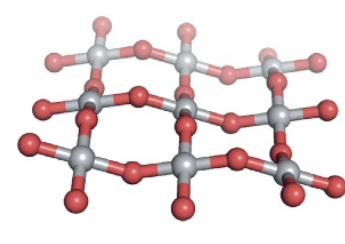

Figure 1 | Correlated disorder in square ice analogues. (a) A configurational fragment of square ice. The water molecules are arranged on a square lattice and are oriented so as to satisfy local hydrogen-bonding rules: each molecule accepts two hydrogen bonds and donates two hydrogen bonds. There is no unique solution to satisfying these local constraints, and so the square ice state is configurationally disordered. (b) A square-planar transition-metal cyanide configuration that maps onto the ice-like state in a. Here each metal cation (coloured green) is coordinated by two nitrogen atoms (blue) and two carbon atoms (grey) such that each $\mathrm{N}$ atom is opposite to a $\mathrm{C}$ atom. (c) Transition metal oxynitrides adopt a related structure ${ }^{14}$, in which square-grid layers consist of metal cations coordinated by two nitrogen atoms (blue) and two oxygen atoms (red). The topological equivalence to the square ice configuration can be seen by alternately considering $\mathrm{O}-\mathrm{M}-\mathrm{O}$ and $\mathrm{N}-\mathrm{M}-\mathrm{N}$ orientations for neighbouring metal centres (shaded regions) ${ }^{26}$. (d) Displacive modulation of a square $\mathrm{MO}_{2}$ lattice by in-plane $\left[\mathrm{MO}_{4}\right]$ rotations gives configurations that again map onto the square ice state. The correspondence relates displacements above and below the plane with, respectively, $\mathrm{O}$ and $\mathrm{N}$ atoms of the oxynitride configuration shown in $\mathbf{c}$.

for disorder is encoded in the combination of the symmetry of the water molecule (that is, the structural building block) and the lattice on which the water molecules are arranged (here enforced by the directionality of the chemical interactions between building blocks). Any system that shares these geometric features will be characterized by the same configurational degeneracy. So, for example, replacing $\mathrm{O}-\mathrm{H} \ldots \mathrm{O}$ linkages by the $\mathrm{M}-\mathrm{C}-\mathrm{N}-\mathrm{M}$ motif found in transition-metal cyanides gives a mapping that-in 3D-relates head-to-tail cyanide disorder in $\mathrm{Cd}(\mathrm{CN})_{2}$ to water molecule orientations in cubic ice (Fig. 1b) ${ }^{13}$. The question of $\mathrm{O} / \mathrm{N}$ ordering within square grid layers of transition-metal oxynitrides presents a related problem, which maps onto the square ice model following geometry inversion from one site to the next (Fig. 1c) ${ }^{14}$. These examples involve compositional or orientational modulations of the square lattice, but the same ideas are well-known to translate to a variety of modulation types, many of which are key to material function: for example; displacive, electronic, charge density, spin density, orbital and spin orientation (Fig. 1d). For ice-like disorder on the diamond lattice, these mappings are well-established in the literature: hence the 'Coulomb phases ${ }^{15}$ of charge ${ }^{16}$, orbital ${ }^{17}$ and $\operatorname{spin}^{18}$ ices.

In seeking to generalise ice-like states, we take our lead from the reticular chemistry approach for generating network 
structures ${ }^{19,20}$. The key idea here is that lattice topologies can be considered in terms of the assembly of nodes and linkers (Fig. 2a): the geometry of the node determines the possible topologies of the corresponding lattice. In this context, the various square-ice systems of Fig. 1 can be considered perturbations of the square lattice in which two adjacent linkers are distinguished among the four that meet at each square node. If we identify three linkers rather than two, then we generate a distinct family of disordered configurations that-from a reticular chemistry perspectivemight be considered to arise from the linking of T-shaped building blocks (Fig. 2b). As for the square ices, there are many possible realizations of this same state: one mapping is to Anderson's resonance valence bond (RVB) description of singlet pair formation in cuprate superconductors ${ }^{21}$; another is to so-called 'domino' tilings of the plane ${ }^{22}$.

There are in total just six cases to be considered for perturbations of the square lattice. Two of these are trivial (distinguishing either four or zero linkers); two are related to one another (distinguishing one linker being the same as distinguishing three); and the two cases that remain distinguish different pairings of the four linkers, as shown in Fig. 2c,d. We have already met the first of these cases in the guise of square ice (Fig. 1c); the second case-in which the linkers distinguished are opposite one another-is ordered and results in symmetry breaking of the underlying square lattice. So there is a nontrivial relationship between perturbations of the node symmetry and the resulting configurational degeneracy.

Ice-like configurations are not confined to perturbations of the square lattice. Equivalent states for the hexagonal, triangular, diamond, cubic and pyrochlore nets are enumerated in Fig. 2e-s and Supplementary Figs 1-37. The extent of disorder can be deduced from the corresponding diffraction patterns, which contain structured diffuse scattering in cases where there is strong correlated disorder (Fig. 2; ref. 10). What emerges is that a substantive fraction of these systems admit large configurational entropies, with a complex relationship between node geometry and extent of correlated disorder. So as to provide insight into this relationship, we generalise Pauling's approximation for the configurational entropy of ice ${ }^{12,14}$ :

$$
S_{\text {config }} \simeq R \ln \left(\frac{n}{2^{d / 2}}\right) .
$$

a
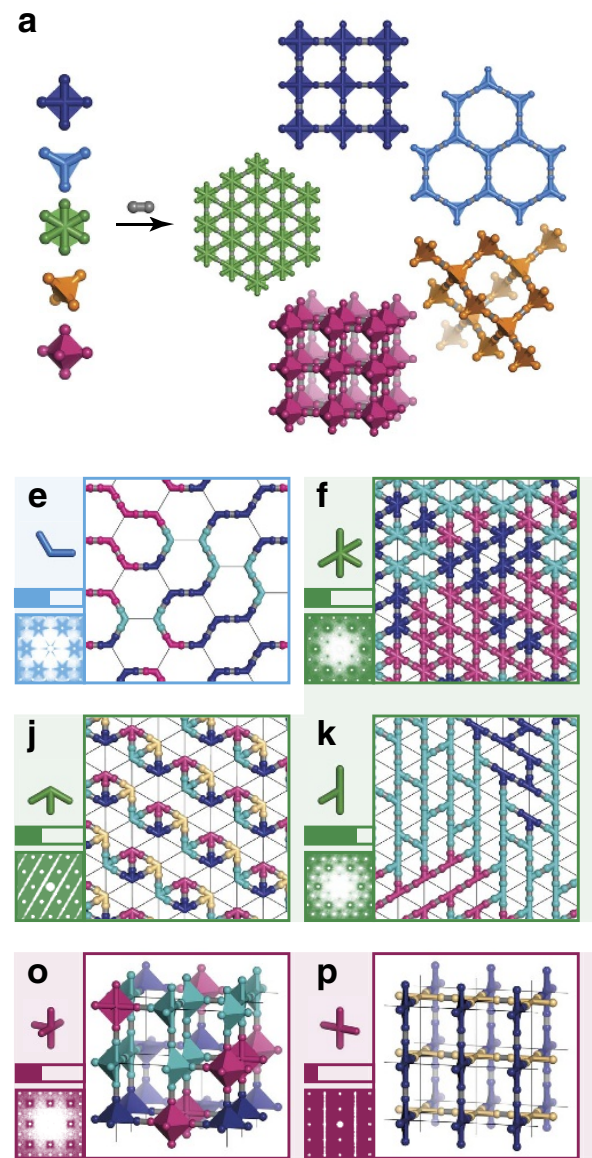
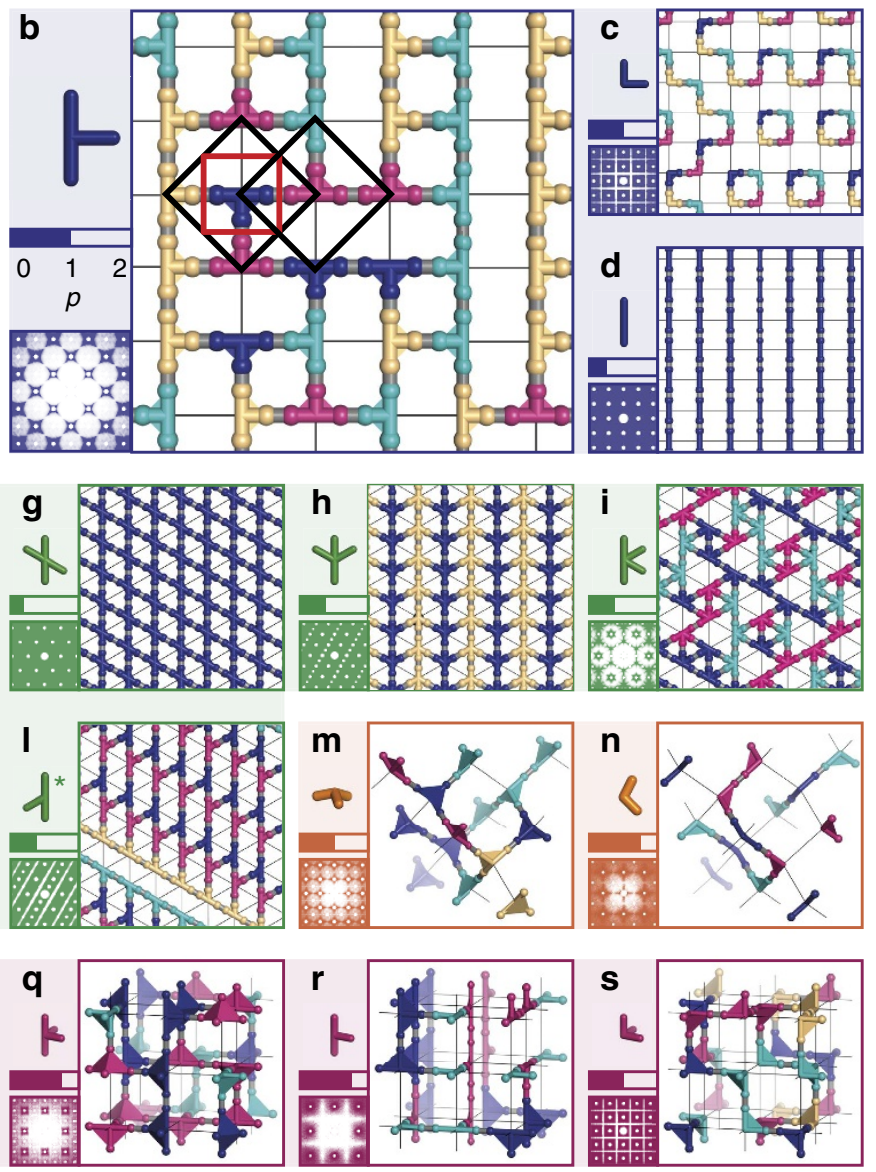

Figure 2 | Reticular design approach to generating procrystalline networks. (a) High-symmetry building blocks connect to form familiar two- and threedimensional networks: square (indigo), hexagonal (blue), triangular (green), diamondoid (orange) and cubic (red). (b-s) Distinguishing different possible subsets of linkers for these high-symmetry lattices gives a variety of ordered and disordered states, which are grouped here according to the parent lattice. For each panel, the perturbed node geometry is shown in the top-left corner, followed immediately below by a representation of the Pauling number $p$, a qualitative indicator of the propensity for disorder. A representative network configuration is shown as the main image, with nodes coloured according to their orientation. One suitable projection of the corresponding X-ray diffraction pattern is given in the bottom-left corner (see Supplementary Figs 1-37 for further details). For the configuration shown in $\mathbf{b}$ two overlapping neighbourhoods are outlined in black. The Dirichlet-Voronoi cell of the neighbourhood lattice is shown in red; the neighbourhood itself is generated by augmenting this cell to include connected latticed points (see Supplementary Figs 38 and 39 and Supplementary Note 1 for further details). The asterisk in I indicates that a single enantiomer of the node geometry is used (cf. $\mathbf{k}$ ); this node is chiral when constrained to lie in two dimensions. Further discussion, including extension to the pyrochlore lattice, is given in Supplementary Tables 2 and 3 and Supplementary Note 2. 
Here $d$ represents the underlying node connectivity and $n$ corresponds to the number of symmetry-equivalent node perturbations of a given type. The value of $n$ can often be determined by inspection, but it is given more rigorously by the ratio of the orders of the point groups of the parent and perturbed node geometries; for example, $n=\left|D_{4 h}\right| /\left|C_{2 v}\right|=4$ for square ice. We call $p=n / 2^{d / 2}$ the Pauling number, with the significance that maximising $p$ maximises the propensity for disorder. In this way one expects low-symmetry perturbations of high-symmetry lattices to lead to states of the greatest configurational entropy, a qualitative relationship that is borne out in practice (Fig. 2). From a materials design perspective, what we are saying is that building block geometry and the arrangement of the interactions between building blocks can together encode for specific types of correlated structural disorder.

The procrystalline state. Common to many of the configurations of Fig. 2 is the absence of translational periodicity characteristic of the crystalline state. For a given configuration, every node experiences the same local environment-and hence it is not meaningful to think of these structures as defective in the vernacular sense. Yet there is no unit cell and space group symmetry that properly describes the topological connectivity. We proceed to argue that these systems should not be considered as crystals, but form a separate class of aperiodic solid with its own characteristics. We will use the term 'procrystalline' to describe this state and to emphasise that conventional crystals might be seen as a special case of the definitions that follow.

The procrystalline state is a dense, overlapping packing of identical fundamental structural units (we use the term 'neighbourhoods'), which are positioned periodically but orientationally permuted as permitted by the point symmetry of the neighbourhood geometry. For magnetic systems, these permutations may involve time reversal operations as realized in, for example, the Ising spin ices ${ }^{23}$. In simple cases the neighbourhood corresponds to the Dirichlet-Voronoi cell of the underlying lattice augmented to include neighbouring, correlated lattice points (Fig. 2b; Supplementary Note 1 and Supplementary Figs 38 and 39). Whereas crystals correspond to the special case in which the neighbourhood orientations are themselves periodic, the more general procrystalline state allows for discrete orientational disorder (cf. the continuous orientational degrees of freedom in, for example, plastic crystals and superionics). Any such disorder will always be correlated since neighbourhoods overlap.

Because their underlying neighbourhood lattice is periodic, all procrystals admit a Bragg diffraction pattern and have a welldefined reciprocal lattice. This diffraction pattern can be analysed using conventional crystallographic approaches but doing so yields a structural model in which neighbourhoods are averaged over their different possible orientations and all information regarding orientational correlation is lost; for example, the states represented in Fig 2r,s share identical Bragg diffraction patterns in spite of their distinct local symmetries. Like crystals, procrystalline phases are characterized by macroscopic point symmetry that can be as high as that of the neighbourhood lattice. Yet, unlike crystals, they can support a complete absence of any point or translational symmetry at the microscopic level. It is the existence of a periodic $3 \mathrm{D}$ reciprocal lattice that distinguishes procrystals from incommensurate and quasicrystalline phases, and which also guarantees a well-defined Brillouin zone and Bloch-wave-like description of phonon and electronic states.

Physical realizations of procrystallinity. The structures of a number of well- and lesser-known materials can be thought of in precisely these terms. This is true by construction for any phase with ice-like disorder; in addition to the various systems described above, the family of ferroelectric phases related to $\mathrm{KH}_{2} \mathrm{PO}_{4}$ is an obvious additional example ${ }^{24}$. Similarly well-established are the statistical mechanical models of $\mathrm{RVB}^{25}$ and loop ${ }^{26}$ states, which correspond to procrystalline lattices with, respectively, one and two linkers distinguished for each node. Hence, physical realizations of either class also fall under our definition (for example, $\mathrm{TaS}_{2}$ (ref. 27) and $\mathrm{SrTaO}_{2} \mathrm{~N}$ (ref. 28)). A less obvious example is the assembly of $p$-terphenyl-3,5,3', $5^{\prime}$-tetracarboxylic acid molecules on pyrolytic graphite to form a hydrogen-bonded network related to the procrystalline lattice illustrated in Fig. 2e (Fig. 3a,b) ${ }^{29}$. This arrangement maps onto the so-called 'rhombus' or 'lozenge' tiling, which in turn corresponds at once to both the Ising triangular antiferromagnet and the RVB description of $\pi$-bonding in graphene $e^{30-32}$. These equivalences are straightforwardly seen in reciprocal space: Fourier transform of the scanning tunnelling microscopy image of Fig. $3 b$ reveals the same distribution of diffuse scattering and 'pinch point' features expected from our simple geometric model (Fig. 3c,d). A further example is the pattern of correlated $\mathrm{Nb}$ offcentre displacements found in the high-temperature cubic phase of $\mathrm{KNbO}_{3}$ (ref. 33). Here the mapping is to the procrystalline lattice illustrated in Fig. $2 \mathrm{~s}$, as reflected (again) in the diffuse scattering distribution observed in single-crystal diffraction measurements (Fig. 3e-g).

We expect the link between characteristic diffuse scattering patterns and particular procrystalline states to aid in diagnosing and understanding a range of problems of correlated disorder ${ }^{10,34}$. For powder diffraction measurements, often the only signature of this diffuse scattering is the presence of $h \mathrm{kl}$ dependent anisotropic peak shape broadening. This is the case, for example, in the scattering patterns of $\mathrm{Pd}(\mathrm{CN})_{2}$ and $\mathrm{Pt}(\mathrm{CN})_{2}$; a procrystalline structural model based on connected square-planar $\left[\mathrm{M}(\mathrm{C} / \mathrm{N})_{4}\right]$ units provides the first convincing description of their diffraction behaviour (Fig. $3 \mathrm{~h}, \mathrm{i})^{35}$. In other cases, procrystalline states (if not necessarily recognized as such) have been inferred from the combination of a disordered average structure and clear signatures of local distortions that can persist only if suitably correlated. Examples include Jahn Teller distortions in the hightemperature orbital-disordered phase of $\mathrm{LaMnO}_{3}$ (refs 36,37) and the high-pressure amorphous phase of $\mathrm{ZrW}_{2} \mathrm{O}_{8}$ (ref. 38). So there is good evidence that a variety of procrystalline phases do exist, even if their structures are difficult to interpret using established crystallographic approaches.

Disorder-phonon coupling. But what of the link between correlated structural disorder and function? In principle, the existence of a well-defined Brillouin zone allows coupling between the structural modulations that characterize the procrystalline state and other physical properties that depend on periodicityfor example, the lattice dynamics and electronic band structure ${ }^{39}$. We tested for coupling of this type using as our example a twodimensional oxynitride lattice (Fig. 1c). The idea was to set up a simple harmonic lattice dynamical model in which we assigned different equilibrium values and stiffnesses to $\mathrm{M}-\mathrm{O}$ and $\mathrm{M}-\mathrm{N}$ bond lengths, and/or to $\mathrm{O}-\mathrm{M}-\mathrm{O}, \mathrm{N}-\mathrm{M}-\mathrm{O}$ and $\mathrm{N}-\mathrm{M}-\mathrm{N}$ bond angles, and then to determine the extent to which correlated compositional order might affect the phonon spectrum. Conventional lattice dynamical calculations are designed for periodic (crystalline) structures, and so we made use of a supercell lattice dynamical approach in order to treat compositional disorder explicitly. We first benchmarked our calculations by determining the 'mean-field' phonon dispersion expected for an average of the different force constant values; we found perfect agreement between our supercell lattice dynamical calculations 
and those obtained using a conventional implementation of the general utility lattice programme (GULP) ${ }^{40}$ (Fig. 4a, Supplementary Fig. 40 and Supplementary Methods 1). For random distributions of equal numbers of $\mathrm{O}$ and $\mathrm{N}$ atoms, the basic phonon structure was similar to the mean-field case, with a slight broadening of phonon frequencies throughout the Brillouin Zone (Fig. 4b). This result is in agreement with ab initio molecular dynamics studies of the related problem of the lattice dynamics of configurational glasses ${ }^{41}$. By contrast, for the procrystalline arrangement there was a dramatic dispersion in energy of the acoustic branches that we observed most noticeably for wave-vectors near the zone corner (Fig. 4c,d). The qualitative similarity to the 'waterfall' phonons observed in thermoelectrics and relaxor ferroelectrics is striking, and suggests a plausible origin for the phenomenon in those systems ${ }^{42,43}$.

So this is our key result: strongly correlated structural disorder allows selective control over physical properties that depend on periodicity. The implication for systems where thermal and electronic conductivities are mediated by, respectively, phonons and electronic states localised in different regions of the Brillouin zone is that disorder-phonon coupling offers a means of selectively reducing thermal conductivity (inversely proportional to phonon bandwidth) without affecting charge transport behaviour. This is an attractive design strategy for developing next-generation thermoelectrics, and one that contrasts with the
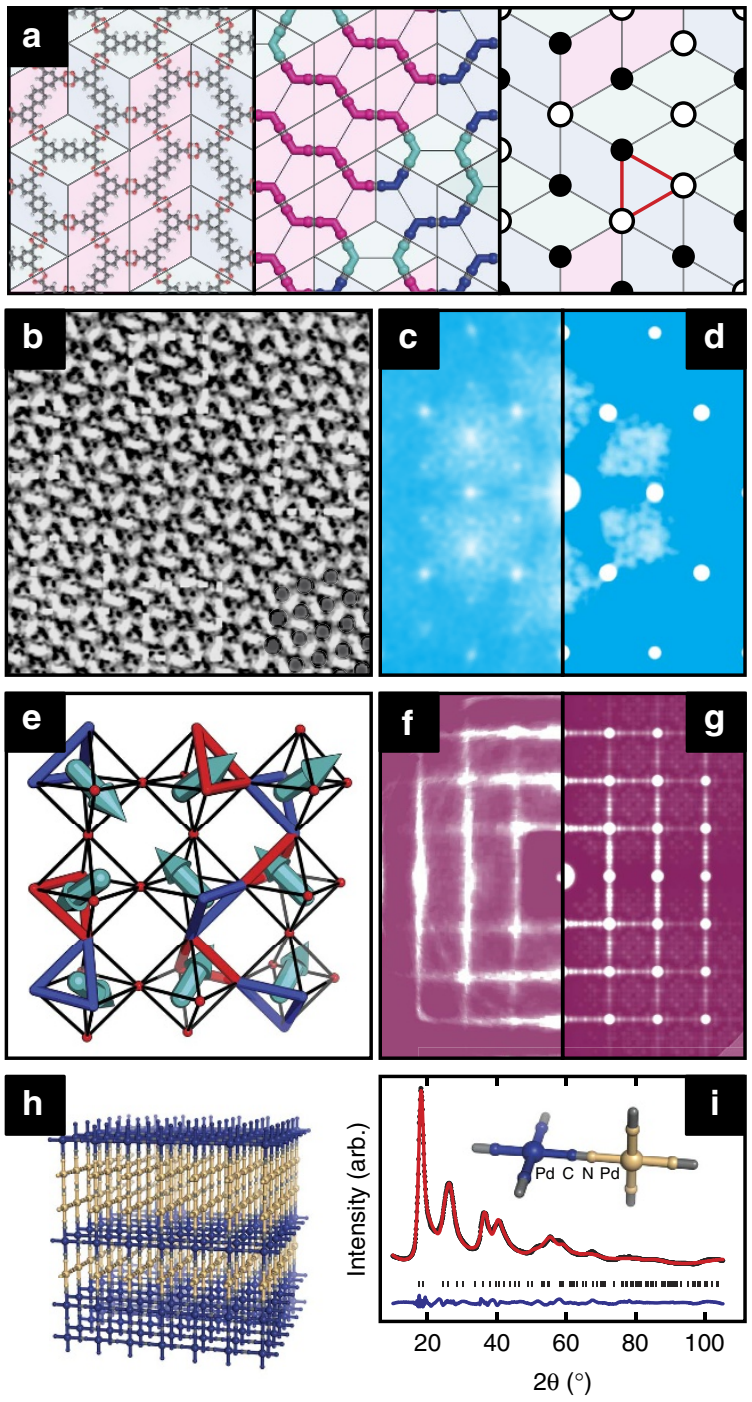

use of 'rattlers' which are indiscriminate in their $\mathbf{k}$-space coupling ${ }^{44}$.

\section{Discussion}

Our reticular chemistry methodology suggests a number of synthetic routes for realising new classes of functional procrystalline solids. Metal-organic frameworks are an obvious platform, given they offer the requisite control over building unit geometry and their energetics tend to be dominated by local interactions ${ }^{45,46}$. While there is reduced scope for coupling between structural disorder and electronic behaviour in these systems, porosity percolation will certainly be affected by disorder and may in turn govern sorption, mechanical and ion storage properties ${ }^{47,48}$. In more conventional inorganics, local symmetry lowering can be achieved by covalency effects (as in mixed-anion perovskites) or by first- or second-order Jahn Teller distortion (as in the chalcogenide thermoelectrics and perovskite ferroelectrics). Moreover, because our analysis is essentially geometric in nature, there is clear scope to extend these concepts to magnetic or electronic states, or indeed to the macroscopic scale. The recent demonstration that disordered metamaterials can show strong structural coupling to light scattering processes is an example relevant to the generation of modern photonics ${ }^{49}$. Thinking beyond the ground-state properties of procrystals, we anticipate the existence of novel collective and hidden degrees of freedom that promise a rich physics of their own; for example, topological excitations ${ }^{50}$ and/or 'hidden order' transitions between distinct local symmetries $^{51}$.

Figure 3 | Representative physical realizations of procrystalline states. (a) Molecules of $p$-terphenyl-3,5, $3^{\prime}, 5^{\prime}$-tetracarboxylic acid (TPTC) self assemble on pyrolytic graphite to form a hexagonal procrystalline network related to that represented in Fig. 2e (ref. 29). One possible arrangement is shown here (left), together with our topological abstraction (centre); molecule orientations in the former map onto the 'missing' linkers of the latter. Shown in background is the configurationally-equivalent rhombus tiling, which further relates to the triangular Ising antiferromagnet (right): opposite vertices of each rhombus are decorated with pairs of 'spin-up' (white circles) and 'spin-down' (black circles) states such that each triplet of neighbouring spins (red triangle) contains at least one state of each kind. (b) A scanning tunnelling microscopy image of the corresponding experimental state. Adapted from ref. 29. Reprinted with permission from AAAS. (c) The Fourier transform of the image in $\mathbf{b}$ showing regions of structured diffuse scattering. (d) The strikingly similar scattering pattern calculated for the hexagonal procrystalline network of Fig. 2e. (e) In the high-temperature cubic phase of $\mathrm{KNbO}_{3}, \mathrm{Nb}^{5+}$ ions displace towards one face of their octahedral coordination environment such that neighbouring $\mathrm{Nb}$ centres displace in the same sense relative to the vector joining the pair. Octahedral faces alternately near to (red) and away from (blue) the displaced $\mathrm{Nb}$ centres gives a procrystalline net related to that shown in Fig. 2s. (f) Structured diffuse scattering observed in single-crystal X-ray diffraction measurements of $\mathrm{KNbO}_{3}$. Adapted from ref. 33. Reprinted with permission of the International Union of Crystallography. (g) Diffuse scattering calculated for the cubic procrystalline network of Fig. 2s. (h) A procrystalline model for the structure of $\mathrm{Pd}(\mathrm{CN})_{2}$ and $\mathrm{Pt}(\mathrm{CN})_{2}$ based on the state represented in Fig. 2p: the two possible orientations of square-planar $\mathrm{M}(\mathrm{C} / \mathrm{N})_{4}$ nodes are shown in indigo and gold. (i) Comparison of the Rietveld fit for this structural model of $\mathrm{Pd}(\mathrm{CN})_{2}$ (red lines) and the experimental X-ray powder diffraction data of ref. 35 (black points, $\lambda=1.54 \AA$ ); tick marks indicate the positions of parent Bragg reflections and the difference (data-fit) is shown in blue (see Supplementary Fig. 42, Supplementary Tables 4 and 5, Supplementary Note 3 and Supplementary Method 2 for further discussion). 


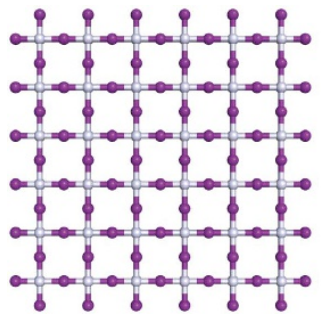

Mean-field

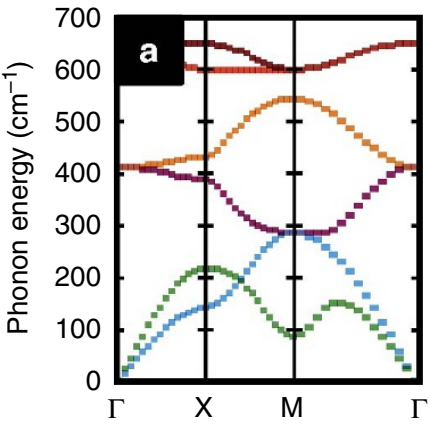

Wave-vector

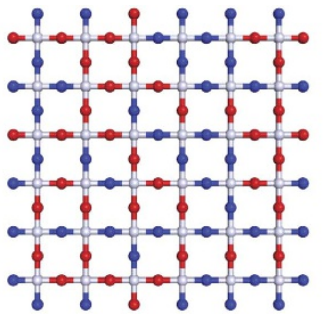

Random disorder

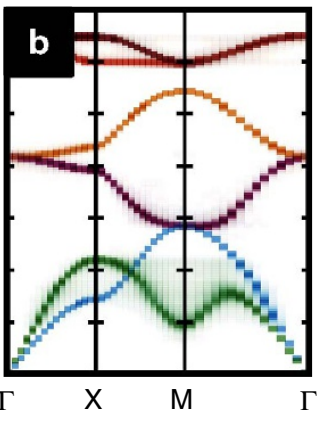

Wave-vector

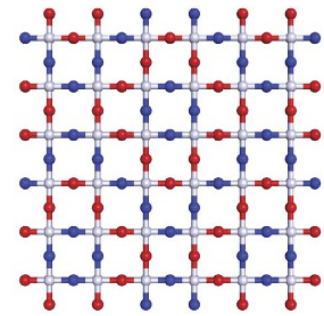

Correlated disorder

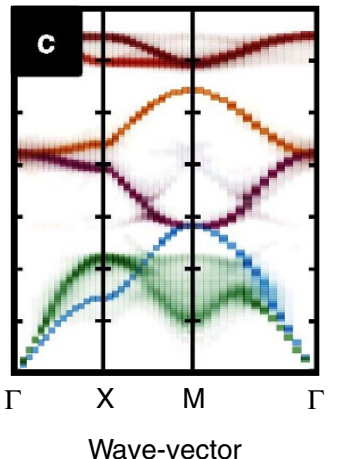

Figure 4 | Selective disorder-phonon coupling in a procrystalline network. (a) Mean-field phonon dispersion curves for a two-dimensional oxynitride lattice, determined using the SCLD approach described in the text. (b) The phonon dispersion determined using SCLD for configurations in which equal numbers of $\mathrm{O}$ and $\mathrm{N}$ atoms are distributed randomly across the $\mathrm{O} / \mathrm{N}$ sites of the same oxynitride lattice. There is a slight broadening of phonon frequencies relative to (a). (c) A third set of phonon dispersion curves, again determined using SCLD but for configurations in which $\mathrm{O}$ and $\mathrm{N}$ atoms have been distributed according to the correlated disorder (procrystalline) model of Fig. 2c. There is now substantive 'waterfall'-like phonon broadening around the M point. (d) The calculated single crystal scattering pattern for the procrystalline model of Fig. 2c, demonstrating that this particular state is characterized by modulations that-like the phonon broadening-are localised around the M point of the BZ. BZ, Brillouin zone; SCLD, supercell lattice dynamics.

\section{Methods}

Procrystal lattice generation. Representative procrystalline configurations were generated using a suite of custom Monte Carlo codes. For each given lattice type, a supercell of the corresponding crystallographic cell was generated and periodic boundary conditions were applied [Supplementary Table 1]. Linkers were randomly assigned one of two initial states $e= \pm 1$. A fictitious configurational energy of typical form:

$$
E=\sum_{i \in \text { nodes }}\left|\left(\sum_{j \in \text { linkers }} e_{i j}\right)-\bar{e}\right|^{2}
$$

was calculated, where $e_{i j}$ represents the state of the $j^{\text {th }}$ linker of the $i^{\text {th }}$ node and the expectation value $\bar{e}$ is a function of the particular node geometry of interest. By way of example, $\bar{e}=0$ for ice-like states on the diamond lattice, since a local '2-in-2-out' configuration is described by two linkers with $e=+1$ and two with $e=-1$. The form of equation (2) is more complex for some combinations of lattice and node geometry, but the relationship that $E=0$ if and only if every node adopts the same local geometry was maintained throughout our study. Monte Carlo minimisation proceeded via the usual Metropolis algorithm ${ }^{52}$, with moves $e_{i j} \rightarrow-e_{i j}$. Because we are interested in defect-free procrystalline states, and because the absolute value of the energy term in equation (2) is not physically meaningful, we terminated our Monte Carlo minimisation not at equilibrium but only when $E=0$. We note that in physical realizations of procrystalline states higher-order correlations may bias towards specific subsets of the $E=0$ configurational space explored in this first-order Monte Carlo approach. All relevant code is available by request.

Diffuse scattering calculations. Physical realizations of the various procrystalline networks generated as described above were produced by placing $\mathrm{Nb}$ atoms at every node position and $\mathrm{O}$ atoms at those linker sites for which $e_{i j}=+1$. Linker sites for which $e_{i j}=-1$ were left vacant. Powder and single crystal X-ray diffraction patterns were generated from individual atomic configurations using the programs CrystalDiffract and SingleCrystal, respectively. Note that the diffuse scattering evident in our diffraction patterns contains contributions only from the linker sites, and its intensity is proportional to the difference in scattering power for $e_{i j}= \pm 1$ occupancies. Consequently, all simple substitutional realizations of a given procrystalline state share the same diffuse scattering pattern, up to a constant factor.
Lattice dynamical calculations. Phonon calculations made use of the GULP program ${ }^{40}$. Supercells of a fictitious two-dimensional NbON square lattice containing $30 \times 30 \times 1$ unit cells (space group symmetry $P m$; relative atom coordinates $\left.\mathrm{Nb}\left(\frac{1}{2}, \frac{1}{2}, 0\right), \mathrm{O} / \mathrm{N} 1\left(\frac{1}{2}, 0,0\right), \mathrm{O} / \mathrm{N} 2\left(0, \frac{1}{2}, 0\right)\right)$ were constructed in three ways. First, a 'mean-field' configuration was generated in which all $\mathrm{O} / \mathrm{N}$ sites were treated as hybrid atoms. Second, random assignment of $\mathrm{O} / \mathrm{N}$ sites to equal numbers of $\mathrm{O}$ and $\mathrm{N}$ atoms gave a 'random disorder' configuration. And, third, a suitable procrystalline configuration of the type illustrated in Fig. 1c was used to assign O and $\mathrm{N}$ atoms such that each $\mathrm{Nb}$ centre was coordinated by exactly two $\mathrm{O}$ and two $\mathrm{N}$ atoms in a cis arrangement; we refer to this as the 'correlated disorder' configuration. In all cases the same set of simple harmonic potentials was used to calculate the lattice enthalpy

$$
E_{\text {latt }}=\frac{1}{2} k_{1} \sum_{i j}\left(r_{i j}-r_{\mathrm{e}}\right)^{2}+\frac{1}{2} k_{2} \sum_{i j k}\left(\theta_{i j k}-\theta_{\mathrm{e}}\right)^{2}+k_{3} \sum_{i j k}\left(1+\cos \theta_{i j k}\right)^{2}
$$

Here $k_{1}=10 \mathrm{eV} \AA^{-2}$ is the stiffness of the $\mathrm{Nb}-\mathrm{O} / \mathrm{N}$ bond, $k_{2}=2 \mathrm{eV} \mathrm{rad}^{-2}$ is the stiffness of the $\mathrm{O} / \mathrm{N}-\mathrm{Nb}-\mathrm{O} / \mathrm{N}$ angle, and $k_{3}=0.1 \mathrm{eV}$ is the stiffness of the $\mathrm{Nb}-\mathrm{O} / \mathrm{N}-\mathrm{Nb}$ angle. This potential model was chosen because it gave a phonon spectrum with physically sensible mode frequencies, good separation between branches, and realistic low-energy mode features (e.g., low-energy tilts at the zone corner). Ultimately, we found that the difference between the phonon dispersion for random and correlated disorder configurations was sensitive to variation in any or all of the $k_{i}, r_{\mathrm{e}}, \theta_{\mathrm{e}}$ and atomic masses for $\mathrm{O}$ and $\mathrm{N}$ atoms. For the phonon dispersion curves illustrated in Fig. 4 we used the very simplest case in which only the value of $\theta_{\mathrm{e}}$ was distinguished: specifically, we used values of $75^{\circ}, 90^{\circ}$ and $105^{\circ}$ for $\mathrm{N}-\mathrm{Nb}-\mathrm{N}, \mathrm{N}-\mathrm{Nb}-\mathrm{O}$ and $\mathrm{O}-\mathrm{Nb}-\mathrm{O}$ angles in each of the random and correlated disorder configurations and a common value of $\theta_{\mathrm{e}}=90^{\circ}$ for the mean-field configuration. In all cases, the same equilibrium bond length $r_{\mathrm{e}}=1 \AA$ was used, corresponding to half the $\mathrm{Nb} \ldots \mathrm{Nb}$ separation in the configurations. Likewise all $\mathrm{O}$ and $\mathrm{N}$ atoms were assigned the same effective mass of 15 a.m.u.

Atomic coordinates were relaxed and phonon calculations performed at the $\Gamma$ point $\left(\mathbf{k}_{\text {supercell }}=(0,0,0)\right)$ of the supercell which corresponds to the $\mathbf{k}$-vector grid of $\frac{1}{30}$ in the reduced mean-field unit cell. The normal modes were calculated as eigenvalues of the dynamical matrix. Despite nominal Pm symmetry, not all of the phonons with atomic displacements along $z$ could be fully separated from in-plane displacements (most probably due to numerical errors). Thus the eigenvalues were calculated using the 'scipy' python library from the two-dimensional component of dynamical matrix which calculated by the GULP. The contribution of each 
eigenvector $\mathbf{e}_{i}$ to all possible $\mathbf{k}$-points was calculated by projecting the eigenvectors at each wave-vector $\mathbf{k}$ in the following way:

$$
\left.e_{i}\right|_{\mathbf{k}}=\sum_{j \alpha}\left|\sum_{\ell} e_{i}(j \ell, \alpha) \exp [i \mathbf{k} \cdot \mathbf{r}(\ell)]\right|^{2},
$$

where $j$ indexes the atoms in each unit cell $\ell$ of the supercell, and $\alpha \in\{x, y\}$. For the mean-field case the projection gave phonon dispersion curves indistinguishable from those obtained using a conventional single-cell GULP calculation (Supplementary Fig. 40 and Supplementary Methods 2). In the case of the random and correlated disorder configurations, the phonon dispersion curves shown in Fig. 4 represent an average over the results obtained for five independent configurations. All relevant code is available by request.

Optical Fourier transform. The scanning tunnelling microscopy image shown in Fig. 3b was Fourier transformed using the Java applet 'Diffraction and Fourier transform ${ }^{53}$. The input data were converted to grayscale, inverted, and optimized for brightness and contrast; the output data were corrected for sample orientation and symmetrised (plane symmetry $p 6 \mathrm{~m}$ ). The actual image used and its raw transform are shown in Supplementary Fig. 41.

\section{References}

1. Natta, G. \& Nasini, A. G. The crystal structure of xenon. Nature 125, 457 (1930).

2. David, W. I. F. et al. Crystal structure and bonding of ordered $\mathrm{C}_{60}$. Nature 353, 147-149 (1991)

3. Reddy, V. S., Natchiar, K., Stewart, P. L. \& Nemerow, G. R. Crystal structure of human adenovirus at $3.5 \AA$ resolution. Science 329, 1071-1075 (2010).

4. Meier, W. M. Molecular Sieves 10-27 (Soc. of Chem. and Ind., 1968).

5. Eddaoudi, M. et al. Modular chemistry: secondary building units as a basis for the design of highly porous and robust metal-organic carboxylate frameworks. Acc. Chem. Res. 34, 319-330 (2001).

6. Dyer, M. S. et al. Computationally assisted identification of functional inorganic materials. Science 340, 847-852 (2013).

7. Pitcher, M. J. et al. Tilt engineering of spontaneous polarization and magnetization above $300 \mathrm{~K}$ in a bulk layered perovskite. Science 347, 420-424 (2015).

8. Steinhardt, P. J. \& Jeong, H.-C. A simpler approach to Penrose tiling with implications for quasicrystal formation. Nature 382, 431-433 (1996).

9. Damasceno, P. F., Engel, M. \& Glotzer, S. C. Predictive self-assembly of polyhedra into complex structures. Science 337, 453-457 (2012).

10. Keen, D. A. \& Goodwin, A. L. The crystallography of correlated disorder. Nature 521, 303-309 (2015).

11. Algara-Siller, G. et al. Square ice in graphene nanocapillaries. Nature 519, 443-445 (2015).

12. Pauling, L. The structure and entropy of ice and of other crystals with some randomness of atomic arrangement. J. Am. Chem. Soc. 57, 2680-2684 (1935).

13. Fairbank, V. E., Thompson, A. L., Cooper, R. I. \& Goodwin, A. L. Charge-ice dynamics in the negative thermal expansion material $\mathrm{Cd}(\mathrm{CN})_{2}$. Phys. Rev. B 86, 104113 (2012)

14. Camp, P. J., Fuertes, A. \& Attfield, J. P. Sub-extensive entropies and open order in perovskite oxynitrides. J. Am. Chem. Soc. 134, 6762-6766 (2012).

15. Henley, C. L. The 'Coulomb phase' in frustrated systems. Ann. Rev. Cond. Matt. Phys 1, 179-210 (2010).

16. Shoemaker, D. P. et al. Atomic displacements in the charge ice pyrochlore $\mathrm{Bi}_{2} \mathrm{Ti}_{2} \mathrm{O}_{6} \mathrm{O}^{\prime}$ studied by neutron total scattering. Phys. Rev. B 81, 144113 (2010).

17. Chern, G.-W. \& Wu, C. Orbital ice: an exact Coulomb phase on the diamond lattice. Phys. Rev. E 84, 061127 (2011).

18. Bramwell, S. T. \& Gingras, M. J. P. Spin ice state in frustrated magnetic pyrochlore materials. Science 294, 1495-1501 (2001).

19. Wells, A. F. Structural Inorganic Chemistry (Clarendon Press, 1984) 5th edn.

20. Yaghi, O. M. et al. Reticular synthesis and the design of new materials. Nature 423, 705-714 (2003).

21. Anderson, $\mathrm{P}$. W. The resonating valence bond state in $\mathrm{La}_{2} \mathrm{CuO}_{4}$. Science 235, 1196-1198 (1987).

22. Kasteleyn, P. W. The statistics of dimers on a lattice. I. The number of dimer arrangements on a quadratic lattice. Physica 27, 1209-1225 (1961).

23. Bramwell, S. T. \& Harris, M. J. Frustration in Ising-type spin models on the pyrochlore lattice. J. Phys. Cond. Matt. 10, L215-L220 (1998).

24. Slater, J. C. Theory of the transition in $\mathrm{KH}_{2} \mathrm{PO}_{4}$. J. Chem. Phys. 9, 16-33 (1941).

25. Anderson, P. W. Resonating valence bonds: a new kind of insulator? Mater. Res. Bull. 8, 153-160 (1973).

26. Kondev, J. Liouville field theory of fluctuating loops. Phys. Rev. Lett. 78, 4320-4323 (1997).
27. Tosatti, E. \& Fazekas, P. On the nature of the low-temperature phase of 1T-TaS 2 . J. Phys. Coll. 37, 165-168 (1976).

28. Günther, E., Hagenmayer, R. \& Jansen, M. Strukturuntersuchungen an den Oxidnitriden $\mathrm{SrTaO}_{2} \mathrm{~N}, \mathrm{CaTaO}_{2} \mathrm{~N}$ und $\mathrm{LaTaON}_{2}$ mittels Neutronen- und Röntgenbeugung. Z. Anorg. Allg. Chem. 626, 1519-1525 (2000).

29. Blunt, M. O. et al. Random tiling and topological defects in a two-dimensional molecular network. Science 322, 1077-1081 (2008).

30. Wannier, G. H. Antiferromagnetism. The triangular Ising net. Phys. Rev. 79, 357-364 (1950).

31. Fisher, M. E. Statistical mechanics of dimers on a plane lattice. Phys. Rev. 124, 1664-1672 (1961).

32. Kasteleyn, P. W. Dimer statistics and phase transitions. J. Math. Phys. 4, 287-293 (1963).

33. Comès, R., Lambert, M. \& Guinier, A. Désordre linéaire dans les cristaux (cas du silicium, du quartz, et des pérovskites ferroélectriques). Acta Cryst. A 26, 244-254 (1970).

34. Welberry, T. R. \& Weber, T. One hundred years of diffuse scattering. Cryst. Rev. 22, 2-78 (2016).

35. Hibble, S. J. et al. Structures of $\operatorname{Pd}(\mathrm{CN})_{2}$ and $\operatorname{Pt}(\mathrm{CN})_{2}$ : intrinsically nanocrystalline materials? Inorg. Chem. 50, 104-113 (2011).

36. Božin, E. S. et al. Local structural aspects of the orthorhombic to pseudo-cubic phase transformation in $\mathrm{La}_{1-x} \mathrm{Ca}_{x} \mathrm{MnO}_{3}$. Physica $B$ 385-386, 110-112 (2006).

37. Ahmed, M. R. \& Gehring, G. A. Volume collapse in $\mathrm{LaMnO}_{3}$ studied using an anisotropic Potts model. Phys. Rev. B 79, 174106 (2009).

38. Keen, D. A. et al. Structural description of pressure-induced amorphization in $\mathrm{ZrW}_{2} \mathrm{O}_{8}$. Phys. Rev. Lett. 98, 225501 (2007).

39. Valla, T. et al. Anisotropic electron-phonon coupling and dynamical nesting on the graphene sheets in superconducting $\mathrm{CaC}_{6}$ using angle-resolved photoemission spectroscopy. Phys. Rev. Lett. 102, 107007 (2009).

40. Gale, J. D. GULP: a computer program for the symmetry-adapted simulation of solids. J. Chem. Soc., Faraday Trans. 93, 629-637 (1997).

41. Fang, H., Dove, M. T., Rimmer, L. H. N. \& Misquitta, A. J. Simulation study of pressure and temperature dependence of the negative thermal expansion in $\mathrm{Zn}(\mathrm{CN})_{2}$. Phys. Rev. B 88, 104306 (2013).

42. Gehring, P. M., Park, S.-E. \& Shirane, G. Soft phonon anomalies in the relaxor ferroelectric $\mathrm{Pb}\left(\mathrm{Zn}_{1 / 3} \mathrm{Nb}_{2 / 3}\right)_{0.92} \mathrm{Ti}_{0.08} \mathrm{O}_{3}$. Phys. Rev. Lett. 84, 5216-5219 (2000).

43. Delaire, O. et al. Giant anharmonic phonon scattering in PbTe. Nat. Mater. 10, 614-619 (2011).

44. Christensen, M. et al. Avoided crossing of rattler modes in thermoelectric materials. Nat. Mater. 7, 811-815 (2008).

45. Cairns, A. B. \& Goodwin, A. L. Structural disorder in molecular framework materials. Chem. Soc. Rev. 42, 4881-4893 (2013).

46. Cliffe, M. J. et al. Correlated defect nanoregions in a metal-organic framework. Nat. Commun. 5, 4176 (2014).

47. Wessells, C. D., Huggins, R. A. \& Cui, Y. Copper hexacyanoferrate battery electrodes with long cycle life and high power. Nat. Commun. 2, 550 (2011).

48. Cliffe, M. J., Hill, J. A., Murray, C. A., Coudert, F.-X. \& Goodwin, A. L. Defectdependent colossal negative thermal expansion in UiO-66(Hf) metal-organic framework. Phys. Chem. Chem. Phys. 17, 11586-11592 (2015).

49. Riboli, F. et al. Engineering of light confinement in strongly scattering disordered media. Nat. Mater. 13, 720-725 (2014).

50. Bak, P. Phenomenological theory of icosahedral incommensurate ('quasiperiodic') order in Mn-Al alloys. Phys. Rev. Lett. 54, 1517-1519 (1985).

51. Toudic, B. et al. Hidden degrees of freedom in aperiodic materials. Science 319, 69-71 (2008).

52. Metropolis, N., Rosenbluth, A. W., Rosenbluth, M. N., Teller, A. H. \& Teller, E. Equation of state calculations by fast computing machines. J. Chem. Phys. 21, 1087-1092 (1953).

53. Schoeni, N. \& Chapuis, G. Fourier Transform, http://escher.epfl.ch.fft/ (2006).

\section{Acknowledgements}

A.R.O., A.B.C., M.J.C., A.S. and A.L.G. gratefully acknowledge financial support from the EPSRC (Grant EP/G004528/2), the ERC (Grant 279705), from the Diamond Light Source to A.R.O., and from the Leverhulme Trust and Swiss National Science Foundation to A.S. This project has received funding from the European Union's Horizon 2020 research and innovation programme under the Marie Skłodowska-Curie grant agreement No. 641887 (Project Acronym: DEFNET-ETN). We are grateful to U. Waghmare (Bangalore), D.A. Keen (ISIS), W.J.K. Fletcher (Oxford) and J.A.M. Paddison (Georgia Tech.) for relevant discussions.

\section{Author contributions}

All authors contributed to the development of the conceptual basis for describing the procrystalline state. A.R.O. carried out the Monte Carlo simulations and single-crystal diffuse scattering calculations. A.R.O. and A.S. performed the lattice dynamical 
calculations. A.B.C. carried out the powder diffraction refinements. A.R.O., M.G.T. and A.L.G. conceived the study. A.L.G. wrote the paper with input from all authors.

\section{Additional information}

Supplementary Information accompanies this paper at http://www.nature.com/ naturecommunications

Competing financial interests: The authors declare no competing financial interests.

Reprints and permission information is available online at http://npg.nature.com/ reprintsandpermissions/
How to cite this article: Overy, A. R. et al. Design of crystal-like aperiodic solids with selective disorder-phonon coupling. Nat. Commun. 7:10445 doi: 10.1038/ncomms10445 (2016).

(c) (i) This work is licensed under a Creative Commons Attribution 4.0 International License. The images or other third party material in this article are included in the article's Creative Commons license, unless indicated otherwise in the credit line; if the material is not included under the Creative Commons license, users will need to obtain permission from the license holder to reproduce the material. To view a copy of this license, visit http://creativecommons.org/licenses/by/4.0/ 